\title{
1 Ventricular response during lungeing exercise in horses with lone atrial fibrillation
}

2 Tinne Verheyen*, Annelies Decloedt, Nicky Van Der Vekens, U. Stanislas Sys, Dominique 3 De Clercq and Gunther van Loon

4 Department of Large Animal Internal Medicine, Faculty of Veterinary Medicine, Ghent 5 University, Salisburylaan 133, 9820 Merelbeke, Belgium

6 *Corresponding author: Tinne.Verheyen@UGent.be

7 Keywords: horse; cardiology; electrocardiography; R-on-T phenomenon; ectopy; aberrant 8 conduction

9

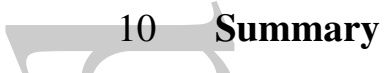

11 Reasons for performing the study: Atrial fibrillation (AF) is the most important dysrhythmia 12 affecting performance in horses and has been associated with incoordination, collapse and 13 sudden death. Limited information is available on ventricular response during exercise in

14 horses with lone AF.

15 Objectives: To investigate ventricular response in horses with lone AF during a standardised 16 lungeing exercise test.

17 Methods: A modified base-apex electrocardiogram was recorded at rest and during a 18 standardised lungeing exercise test from 43 horses diagnosed with lone AF. During the test 19 horses walked for $7 \mathrm{~min}$, trotted for $10 \mathrm{~min}$, cantered for $4 \mathrm{~min}$, galloped one minute and 20 recovered for $7 \mathrm{~min}$.

This article has been accepted for publication and undergone full peer review but has not been through the copyediting, typesetting, pagination and proofreading process, which may lead to differences between this version and the Version of Record. Please cite this article as doi: 10.1111/j.2042-3306.2012.00653.x 
21 Results: Individual average heart rate during walk ranged from 42 to $175 \mathrm{bpm}$, during trot 22 from 89 to $207 \mathrm{bpm}$, during canter from 141 to $269 \mathrm{bpm}$, and during gallop from 191 to 311 23 bpm. Individual beat-to-beat maximal heart rate ranged from 248 to $492 \mathrm{bpm}$. Ventricular 24 premature depolarisations were present in $81 \%$ of the horses: at rest (16\%), during exercise 25 (69\%), and during recovery (2\%). In $33 \%$ of the horses, broad QRS complexes with R-on-T 26 morphology were found.

27 Conclusions: Exercising horses with lone AF frequently develop disproportionate tachycardia. 28 In addition, QRS broadening and even R-on-T morphology is frequently found. QRS

29 broadening may originate from ventricular ectopic foci or from aberrant intra-ventricular 30 conduction, for example due to bundle branch block. This might explain the high number of 31 complexes currently classified as ventricular premature depolarisations.

32 Potential relevance: Prevalence of QRS broadening and especially R-on-T was very high in 33 horses with AF and was found at low levels of exercise. These dysrhythmias are considered 34 risk factors for the development of ventricular tachycardia and fibrillation and they might 35 explain signs of weakness, collapse or sudden death that have been reported in horses with 36 AF.

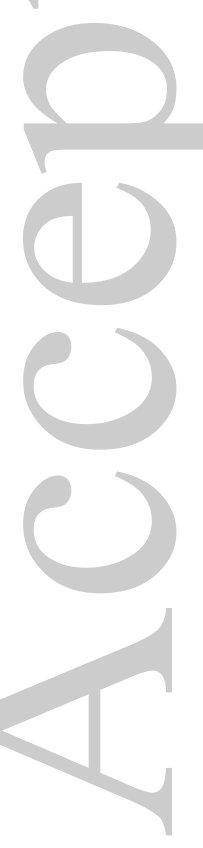




\section{Introduction}

38 With a prevalence of about 2.5\% [1], atrial fibrillation (AF) is the most important

39 dysrhythmia affecting performance in horses. During AF in horses, multiple wavelets

40 propagate through the atria at a rate of approximately 300 to 500 pulses per min [2; 3]. The

41 term lone $\mathrm{AF}$ is used when there is no evidence of underlying structural disease and AF

42 occurs in an otherwise healthy individual. The high parasympathetic tone in horses with lone

43 AF causes the atrioventricular (AV) node to block most of these impulses, resulting in a

44 normal ventricular rate at rest. The chaotic self-sustained electrical activity in the fibrillating

45 atria causes independent activation of individual muscle fibres rather than the synchronous

46 contraction seen during normal sinus rhythm [4]. As a consequence, atrial contribution to

47 ventricular filling is lost, causing a decrease in stroke volume, especially during exercise. In

48 addition, sympathetic tone prevails during exercise, reducing the blocking function of the AV

49 node. This causes many of the atrial fibrillatory impulses to be conducted to the ventricles,

50 resulting in a disproportionate tachycardia. Both factors reduce cardiac function and therefore

51 athletic ability. Depending on the exercise load, AF can be an incidental finding or can result

52 in performance loss or in signs of weakness and incoordination (9\%) or even collapse during

53 work $(2 \%)[5]$.

54 Although numerous studies have been dedicated to AF and in particular its treatment options,

55 limited information is available concerning electrocardiography (ECG) during exercise in 56 horses with AF [6-9]. The aim of this study was to report the ventricular response and 57 dysrhythmias in horses with lone AF during a standardised lungeing exercise test.

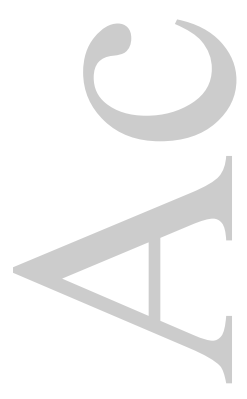




\section{Materials and methods}

\section{Study population}

60 Forty-three horses that were presented at the Department of Large Animal Internal Medicine,

61 Ghent University for cardiac examination and were diagnosed with lone AF were included in 62 this study..

63 Horses (17 mares, 5 stallions, 21 geldings; 39 Warmbloods, one Friesian, one Anglo Arabian,

642 French Trotters) had an age of $10.6 \pm 3.6$ years (mean \pm standard deviation, s.d.) (range 46520 years), a height of $170.2 \pm 6.1 \mathrm{~cm}$ (range $152-181 \mathrm{~cm}$ ) and a body weight of $584.5 \pm 54 ; 1$

$66 \mathrm{~kg}$ (range 468-710 kg). Presumptive AF duration was 3 weeks to one year; in 3 horses AF

67 duration was not known. Horses were used for dressage $(n=7)$, jumping $(n=21)$, both $(\mathrm{n}=$

$683)$, eventing $(n=2)$, recreational $(n=6)$, trotting $(n=2)$, cross-country $(n=1)$ or driving $(n=$

69 1). Presenting signs were performance reduction $(n=28)$, epistaxis $(n=3)$ and weakness and

70 collapse $(n=1)$. Eleven horses showed no signs.

71 In 36 horses, plasma ionised calcium, potassium and magnesium concentrations were 72 determined. Thirty-six horses were successfully converted using transvenous electrical 73 conversion $($ TVEC) $(n=33)$ or quinidine sulphate $(n=3)$. Three horses failed to convert by 74 TVEC. In 4 horses no treatment was initiated.

\section{Electrocardiography}

76 Modified base-apex ECG was performed using a Televet $100^{\circledR}$ recording system $^{\mathrm{a}}$ as described 77 elsewhere [10]. Briefly, 4 self-adhesive electrodes were positioned under a girth ${ }^{\mathrm{b}}$ in a 78 modified base-apex configuration: the right arm electrode was positioned $15 \mathrm{~cm}$ right of the 79 withers, the left leg electrode on the thorax caudal to the left elbow. The left arm electrode 80 was placed $10 \mathrm{~cm}$ above the green one. The reference electrode (right leg) was positioned 15 $81 \mathrm{~cm}$ left from the withers. All electrodes were connected to the recording device in the girth. 
82 The ECG was visualised in real time on a laptop computer and the signal was digitally stored

83 to allow offline analysis.

\section{Exercise protocol}

85 Recording started as soon as the monitoring system was fitted, including a 15 min recording

86 at rest. The exercise protocol was a standardised lungeing exercise test in which horses

87 walked for $7 \mathrm{~min}$, trotted for $10 \mathrm{~min}$, cantered for $4 \mathrm{~min}$, and galloped for one minute. The

88 recovery period was $7 \mathrm{~min}$. The 2 min walk to the exercise ring and back was included in the

89 recording time during walk and the recovery phase, respectively.

90 Intra-atrial electrocardiography

91 In 37 horses, a bipolar temporary pacing electrode ${ }^{c}$ was positioned in the right atrium in the

92 standing horse. This allowed simultaneous recording of an intra-atrial electrogram and a base-

93 apex ECG at rest using a modified Televet $100^{\circledR}$ recorder $^{\mathrm{d}}$ or Pacemaker Programmer. $^{\mathrm{e}}$ The $^{\mathrm{s}}$

94 signal was digitally stored to allow offline analysis.

\section{Data analysis}

96 Offline analysis of exercise ECGs was performed by an experienced observer (T.V.) using

97 dedicated software. ${ }^{\mathrm{f}}$ All recorded ECGs were of diagnostic quality, and $16 \%$ showed 98 important motion artefacts but still allowed accurate diagnosis. Standard gain $(10 \mathrm{~mm} / \mathrm{mV})$

99 and paper speed $(25 \mathrm{~mm} / \mathrm{s})$ were increased up to $20 \mathrm{~mm} / \mathrm{mV}$ and $200 \mathrm{~mm} / \mathrm{s}$ where necessary

100 to allow accurate analysis. The number and type of dysrhythmias were documented by visual

101 inspection. In each horse, the average heart rate at rest, walk, trot, canter and gallop was

102 calculated; maximal heart rate was calculated from the single shortest RR interval obtained

103 during the protocol. The duration of the QRS complexes and S waves was measured for 50

104 consecutive cycles at rest and during galloping, and in QRS complexes with an aberrant

105 morphology (Fig 1). QRS complexes with abnormal morphology were categorised as

106 ventricular premature depolarisations (VPDs), when changes in relative size of $\mathrm{Q}, \mathrm{R}$ or $\mathrm{S}$ 
107 waves leading to changes in morphology of the complex were observed or when duration of 108 the QRS complex was altered. Slight changes in QRS amplitude due to respiration were not 109 taken into account. When the $\mathrm{R}$ wave of the abnormal QRS complex was projected on the $\mathrm{T}$ 110 wave of the previous QRS complex, QRS complexes were categorised as 'R-on-T' 111 complexes.

112 In each horse, measurements of QRS and S-wave duration of normal complexes (rest and 113 gallop), VPDs and R-on-T complexes were averaged over the measured cycles. The resulting 114 means were compared between the complex types by a linear mixed model with complex type 115 as fixed categorical effect and with the horses as subjects in a repeated measurements 116 analysis.

117 Atrial fibrillation cycle length (AFCL) was calculated from intra-atrial electrograms as an 118 estimate of atrial refractoriness by measuring the interval between successive atrial 119 depolarisation waves from a $20 \mathrm{~s}$ window. Individual maximal heart rate was compared to 120 AFCL using Pearson's correlation test. Data are presented as mean \pm s.d.. Significance was 121 set at $\mathrm{P}<0.05$.

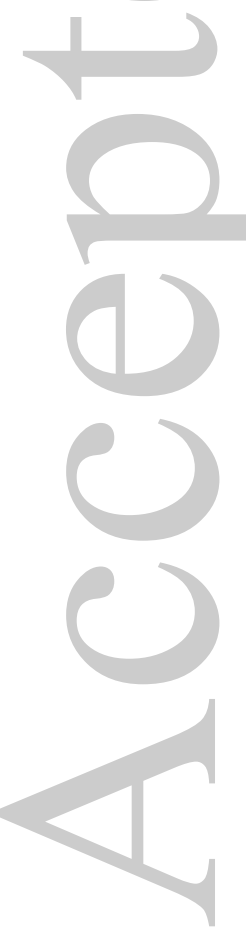




\section{Results}

123 Three horses had mild hypocalcaemia (1.4 mmol/L; reference range $1.5-1.8 \mathrm{mmol} / \mathrm{L})$ and one

124 was both hypocalcaemic $(1 \mathrm{mmol} / \mathrm{L}$; reference range $1.5-1.8 \mathrm{mmol} / \mathrm{L})$ and hypokalaemic $(1.8$

$125 \mathrm{mmol} / \mathrm{L}$; reference range $2.9-4.4 \mathrm{mmol} / \mathrm{L})$.

126 Forty-two horses completed the protocol. In one horse the protocol was terminated during

127 walking to the exercise ring because of a high heart rate (297 bpm at walk). In 2 trotting

128 horses the canter and gallop were replaced by trotting at increased speeds.

129 Individual average heart rates at rest, walk, trot, canter and gallop are shown in Fig 2. At rest

130 and during walk, 35\% of the horses with AF had an average heart rate above the normal

131 reference range (reference range rest: $25-50 \mathrm{bpm}$; reference range walk: 60-80 bpm) [11].

132 During trot and canter the average heart rate was above reference range in $83 \%$ and $98 \%$ of

133 the horses with AF, respectively (reference range trot: 80-120 bpm; canter 120-150 bpm)

134 [11]. During gallop, all horses with AF in this study had an average heart rate above reference 135 range (150-180 bpm) [11]. Individual maximal heart rate during the lungeing exercise test 136 ranged from $248 \mathrm{bpm}$ to $492 \mathrm{bpm}$ (Fig 3), while the normal upper limits in maximal heart rate 137 during vigorous exercise is $240 \mathrm{bpm}$.

138 In $81 \%$ of the horses with AF, QRS complexes with abnormal morphology, categorised as 139 VPDs, were observed at rest (16\%), during exercise $(69 \%)$ or recovery phase (2\%).

140 Encountered abnormal morphologies were RS, rS, S or Rs in type. In $71 \%$ of the horses, 141 different abnormal morphologies were observed. Both at rest and during exercise, abnormal 142 QRS complexes were often associated with episodes of tachycardia due to increased 143 sympathetic tone.

144 In $33 \%$ of the AF horses, broad QRS complexes with an 'R-on-T' morphology were observed 145 (Fig 4). All QRS complexes with R-on-T morphology were associated with increased

146 sympathetic tone: they occurred at rest when horses were aroused, or during fast galloping. 
147 Episodes with R-on-T were short lasting, varying from one beat to 10 consecutive beats.

148 Often R-on-T episodes were terminated by a long RR interval. Number of episodes per horse 149 varied from 1 to 10.

150 Significant QRS shortening occurred during gallop $(\mathrm{P}<0.0005)$. Both VPDs and QRS

151 complexes with R-on-T morphology were significantly longer than normal QRS complexes

152 during gallop $(\mathrm{P}<0.0005)$ and shorter compared to normal QRS complexes at rest $(\mathrm{P}<0.0005)$.

153 R-on-T complexes were not significantly different from VPDs ( $\mathrm{P}=1.0)$ (Fig 5). However, in

154 R-on-T complexes the $\mathrm{R}$ wave is no longer discernible and only the $\mathrm{S}$ wave is measured. $\mathrm{S}$

155 wave duration was significantly longer for R-on-T complexes than for normal complexes at

156 rest $(\mathrm{P}=0.012)$ and during gallop $(\mathrm{P}<0.0005)$. Values for $\mathrm{R}$-on- $\mathrm{T}$ complexes were also

157 significantly longer compared to VPDs $(\mathrm{P}<0.0005)$ (Fig 6).

158 Average AFCL ranged from 128 to 207 ms. In 8 horses the recorded maximal heart rate was 159 slightly higher than atrial fibrillation rate. There was no correlation between calculated AF 160 rate at rest and individual maximal heart rate $(\mathrm{P}=0.591)$

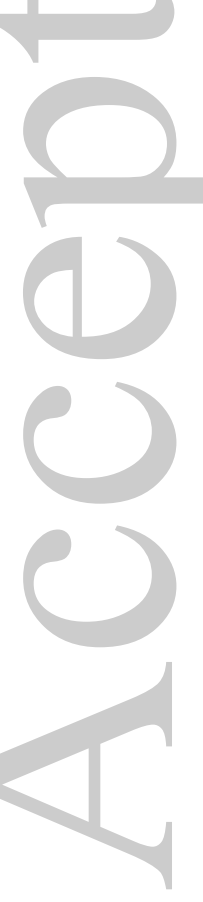




\section{Discussion}

162 This study shows that in horses with lone AF, heart rate can raise high above the normal 163 maximal heart rate. Excessively high heart rates are predominantly present during gallop and 164 when horses were startled. Furthermore, QRS broadening is often found.

165 During AF, the AV node receives a high number of random electrical impulses from multiple

166 wavefronts circulating in the atria. Ventricular response rate is determined by autonomic

167 influences, the amount of concealed conduction and inherent AV nodal function [12; 13]. At

168 rest, parasympathetic tone prevails and causes depressive effects on the AV node,

169 hyperpolarisation and prolonged AV conduction time, which leads to conduction block [14;

170 15]. In this situation, concealed conduction takes place: atrial impulses reach the AV node

171 during the relative refractory phase and hence only partially penetrate into the AV node

172 without reaching the ventricles [16]. Concealed conduction of an impulse affects the

173 conduction of a subsequent impulse by delaying it, blocking it entirely or causing repetitive

174 concealed conduction [17]. It is supposed that during AF, many of the atrial impulses are

175 concealed within the AV node [18]. During exercise or stress however, vagal influence

176 diminishes and sympathetic tone becomes predominant [19]. The refractory period of the AV

177 node shortens, which decreases the occurrence of concealed conduction and can lead to an 178 increase in ventricular rate [20].

179 Another mechanism potentially contributing to increased heart rate during exercise is the 180 dependency of the refractory period of the AV node on cycle length $[21 ; 22]$. The functional 181 refractory period of the AV node shortens slightly with shorter cycle lengths [23; 24], 182 increasing the rate with which atrial impulses can be propagated to the ventricles. Mendez et 183 al. reported the occurrence of 'abnormally' short RR intervals after early atrial premature 184 responses [21]. It seemed that the AV node responded to very early reexcitation with an 185 abrupt shortening of its refractory period, thus leading to very short RR intervals. A possible 
186 explanation for this phenomenon could be a cumulative effect of repeated short cycles on AV

187 nodal refractory period.

188 QRS complexes with abnormal morphology, categorised as VPDs, were observed in $82 \%$ of

189 the horses with $\mathrm{AF}$, with 2 or more different morphologies present in $73 \%$. In $36 \%$ of the

190 horses with AF, QRS complexes with an 'R-on-T' morphology were observed. In

191 comparison, the reported prevalence of VPDs during exercise in clinically healthy dressage

192 and show jumping horses is 5\% [25] and 18\% [26]. In human patients, wide QRS complexes

193 are frequently observed in AF [27]. Two different processes could be causing this broadening:

194 ventricular ectopy or aberrant intra-ventricular conduction of supraventricular impulses [28].

195 Despite the difference in origin of these 2 processes (atrial or ventricular), differentiation is

196 complicated in AF, since the relation between atrial impulses and QRS complexes is never

197 recognisable. The differentiation, however, has prognostic and therapeutic importance, since

198 aberrancy will disappear when sinus rhythm is restored, whereas ventricular ectopy can

199 significantly affect both prognosis and treatment [27; 28].

200 The differentiation is very difficult based on surface electrocardiography alone but in human

201 medicine, several criteria have been suggested amongst which QRS contour and resemblance

202 of the initial deflection of the anomalous complex with that of flanking normal complexes

203 seemed the most useful [28; 29]. Some of the broad QRS complexes in the horses with AF did

204 fulfil the criteria for aberrant conduction. However, it is unknown whether or not these criteria 205 also apply to horses. In 73\% of the horses with AF, abnormal QRS complexes were present 206 during exercise, a period in which sympathetic tone prevails. Sympathetic stimulation 207 accelerates AV nodal conductivity [30] and shortens AV nodal refractoriness. With increasing 208 heart rates, the refractory period of the AV node can become shorter than that of left or right 209 bundle branch, such that atrial impulses conducted through the AV node may hit one of the 210 bundles during its refractory period. When this happens, the impulse is forced to follow an 
211 alternative pathway through the ventricles, leading to aberrant conduction caused by bundle

212 branch block [27]. Twenty percent of the horses had abnormal QRS complexes at rest. In all

213 but one of these horses, the QRS abnormality was observed when horses were distressed or

214 excited, causing increased sympathetic tone and potentially leading to aberrant conduction. In

215 the remaining horse, repeatedly a relatively long RR interval was followed by a short RR

216 interval with altered QRS morphology. This phenomenon is described in human medicine as

217 Ashman phenomenon and is caused by aberrant intra-ventricular conduction due to right

218 bundle branch block. This is explained by the long refractory period of the right bundle

219 branch at slow heart rates compared to the AV node or left bundle branch [27], and the fact

220 that refractory periods are dependent upon the length of the previous RR interval. As such,

221 when a short cycle follows a long one, the right bundle branch with its longer refractory

222 period is still refractory, leading to a QRS complex with a specific aberrant morphology. A

223 similar mechanism was thought to be present in this horse (Fig 7).

224 In many horses, broad QRS complexes had different morphologies, which were thought to be

225 caused by ventricular ectopy. In 4 horses, concomitant hypocalcaemia and/or hypokalaemia

226 was present, which may have contributed to the dysrhythmias [31; 32]. However,

227 abnormalities were mild, and horses without electrolyte disturbances also had ectopic QRS

228 complexes. Abnormal QRS complexes were most frequently observed during periods

229 involving sympathetic stimulation. In human medicine, substantial evidence links enhanced 230 sympathetic activity with ventricular dysrhythmias and sudden cardiac death in patients with 231 various cardiac conditions [33]. Adrenergic facilitation of irregular ventricular activity has 232 been attributed to increased automaticity, decreased diastolic threshold and decreased 233 refractoriness. It has been shown in dog hearts that stimulation of the sympathetic nerves 234 increases temporal dispersion of the refractory periods in ventricular muscle [34]. As a result,

235 re-entry and fractionation of a ventricular wave front can be facilitated. 
236 Many horses with AF had 'R-on-T' morphology on the surface ECG, a term which describes

237 superposition of the ventricular depolarisation of an ectopic ventricular beat on the $\mathrm{T}$ wave of 238 the preceding beat $[35 ; 36]$. In man, R-on- $\mathrm{T}$ is considered a high-grade risk factor for 239 development of ventricular tachycardia or fibrillation [36; 37]. However, in man, atrial 240 fibrillation is not typically associated with R-on-T. Although the morphology of the 241 phenomenon observed in this study in horses with AF seems to be identical to what is 242 described as 'R-on- $\mathrm{T}$ ' in human medicine, it cannot be proven with certainty whether this 243 rhythm was supraventricular or ventricular in origin. A recent study in healthy horses reported 244 on what was called 'torsade-like polymorphic ventricular tachycardia' (T-PMVT) in the 245 immediate post-race period [38]. Although QRS complexes described in that study showed

246 similarities with the 'R-on-T' complexes seen in the current study, they occurred typically

247 during race recovery. The authors also suggested autonomic influences as a potential cause 248 for these dysrhythmias.

249 In human medicine, aberrancy is considered to be of limited clinical significance in AF, 250 whilst VPDs are regarded as potential risk factors for the induction of ventricular tachycardia 251 or fibrillation [28]. It is not known whether this is also the case for horses. Whilst R-on-T 252 occurred relatively frequently, in none of the horses it deteriorated to ventricular fibrillation. 253 This might suggest that R-on- $\mathrm{T}$ is caused by aberrancy rather than ventricular ectopy. 254 However, signs of weakness, collapse and even sudden death have been observed in AF 255 horses [5] and could have been associated with ventricular ectopy.

256 Individual beat-to-beat maximal heart rate was compared to AFCL in order to investigate the 257 origin of the broad QRS complexes. If broad QRS complexes were due to bundle branch 258 block, their origin would be supraventricular, and the shortest RR interval would approximate 259 or be longer than the AFCL. If the shortest RR interval would be much shorter than the 260 AFCL, broad QRS complexes would have to be ventricular in origin. Ten horses had a 
261 maximal heart rate in excess of the atrial fibrillation rate. However, differences were small

262 and could be explained by temporal and spatial dispersion in AFCL and by the fact that 263 increased sympathetic tone shortens AFCL [18]. As such, the exact origin of the abnormal 264 QRS morphology remained unknown.

265 A limitation of the study is the standardised lungeing exercise tests performed in horses with

266 AF, meaning that for many horses workload was below their normal level. Still, a very high

267 prevalence of dysrhythmias was found. Although maximal exercise was not studied, higher

268 workload might be associated with more severe rhythm disturbances.

269 In conclusion, horses with $\mathrm{AF}$ frequently develop disproportionate tachycardia during

270 exercise. QRS broadening and R-on-T phenomenon are often found and may originate from

271 ventricular ectopy or aberrant intra-ventricular conduction. At this point, the origin of broad

272 QRS complexes in horses with AF remains uncertain. The high number of VPDs in these

273 horses might indicate that some of these complexes result from aberrant conduction rather 274 than ventricular ectopy. However, in human medicine, R-on-T is always considered 275 ventricular in origin. QRS broadening and R-on-T complexes might be a risk factor for 276 exercise-associated weakness, collapse or even death. One should be aware of the high 277 prevalence and potential risk factor of these dysrhythmias in horses with lone AF, even if they 278 are used for low level exercise because sudden stress in a resting horse can elicit these 279 dysrhythmias. 280

\section{Authors' declaration of interests}

282 The authors declared no competing interests. 
285 This study was funded by Ghent University.

286

\section{Author contributions}

288 Study design: G. van Loon and T. Verheyen. Data collection and study execution: T. 289 Verheyen, A. Decloedt and N. Van Der Vekens. Data analysis and interpretation: T. Verheyn,

290 A. Decloedt and S.U. Sys. Preparation of the manuscript: T. Verheyen, A. Decloedt, N. Van 291 Der Vekens, D. De Clercq, S.U. Sys and G. van Loon.

292

293 Acknowledgements

294 The authors wish to thank Ing. K. Engel, Engel Engineering Services GmbH, Germany, for 295 adapting the Televet $100^{\circledR}$ device.

296

297 Manufacturers' addresses

$298{ }^{a}$ Televet $100^{\circledR}$, Kruuse, Marslev, Denmark.

299 brthohorse $^{\circledR}$, Mainat Vet, Barcelona, Spain.

300 ' U.S.C.I. Ballinasloe, County Galway, Ireland.

$301{ }^{\mathrm{d}}$ K. Engel, Engel Engineering Services GmbH, Offenbach am Main, Germany.

$302{ }^{\text {e }}$ Programmer 7990, Medtronic, Minneapolis, USA.

$303 \mathrm{f}^{\mathrm{f}}$ Televet $100^{\circledR}$ software version 4.2.0, Kruuse, Marslev, Denmark.

304 


\section{FIGURE LEGENDS}

306 Fig 1: Electrocardiogram showing calliper placement for QRS and S wave duration for a 307 normal complex and S wave duration for a R-on-T complex.

308 Fig 2: Heart rate in 43 horses with lone atrial fibrillation at rest and during exercise. Grey 309 boxes indicate lower quartile to upper quartile with horizontal black line indicating median.

310 Sample minimum and maximum are shown by whiskers. Symbols indicate outliers. Blue bars

311 indicate typical heart rate ranges for each speed in normal horses.

312 Fig 3: Individual beat-to-beat maximal heart rates during exercise as a function of calculated 313 AF rate of 37 horses with lone atrial fibrillation.

314 Fig 4: Electrocardiogram showing R-on-T phenomenon (arrows) in a horse with atrial 315 fibrillation during galloping.

316 Fig 5: Duration (mean \pm s.d.) of normal QRS, abnormal QRS and R-on-T complexes at rest 317 and during exercise. Different letters indicate significant differences.

318 Fig 6: Duration (mean \pm s.d.) of $\mathrm{S}$ waves of normal QRS, abnormal QRS and R-on-T 319 complexes at rest and during exercise. Different letters indicate significant differences.

$320 \quad$ Fig 7: Electrocardiogram showing a long-short cycle with broad QRS complex terminating 321 the sequence, suggestive of Ashman phenomenon. 


\section{References}

323 [1] Else, R.W. and Holmes, J.R. (1971) Pathological changes in atrial fibrillation in the horse. Equine Vet. J. 3, 56-64.

325

326 [2]

327

328

329

$330 \quad[3]$

331

332

333

334 [4]

335

336

337 [5]

338

339

340

341 [6]

342

343

344

345 [7]

346

347

348 [8]

349

350

351 [9]

352

353

$354 \quad[10]$

355

356

357

358

359

360

361

De Clercq, D., van Loon, G., Baert, K., Tavernier, R., Croubels, S., De Backer, P. and Deprez, P. (2006) Intravenous amiodarone treatment in horses with chronic atrial fibrillation. Vet. J. 172, 129-134.

De Clercq, D., Van Loon, G., Baert, K., Tavernier, R., Croubels, S., De Backer, P. and Deprez, P. (2007) Effects of an adapted intravenous amiodarone treatment protocol in horses with atrial fibrillation. Equine Vet. J. 39, 344-349.

Blissitt, K.J. (1999) Diagnosis and treatment of atrial fibrillation. Equine Vet. Educ. 11, 11-19.

Deem, D.A. and Fregin, G.F. (1982) Atrial fibrillation in horses - A review of 106 clinical cases, with consideration of prevalence, clinical signs, and prognosis. J. Am. Vet. Med. Assoc. 180, 261-265.

Gehlen, H., Bubeck, K., Rohn, K. and Stadler, P. (2006) Pulmonary artery wedge pressure during treadmill exercise in warmblood horses with atrial fibrillation. Research in Veterinary Science 81, 134-139.

Deegen, E. and Buntenkötter, S. (1976) Behaviour of the heart rate of horses with auricular fibrillation during exercise and after treatment. Equine Vet. J. 8, 26-29.

Maier-Bock, H. and Ehrlein, H.J. (1978) Heart rate during a defined exercise test in horses with heart and lung diseases. Equine Vet. J. 10, 235-242.

Verheyen, T., Decloedt, A., De Clercq, D., Deprez, P., Sys, S.U. and van Loon, G. (2010) Electrocardiography in horses - part 1: how to make a good recording. Vlaams Diergeneeskundig Tijdschrift 79, 331-336.

Birks, E.K., Durando, M.M. and Martin, B.B.J. (2004) Clinical exercise testing: evaluation of the poor performing athlete. In: Equine Sports Medicine and Surgery Basic and clinical sciences of the equine athlete, Eds: K.W. Hinchcliff, A.J. Kaneps and R.J. Geor, Saunders, Edinburgh. pp 9-18. 
363 [12] Meijler, F.L., Kroneman, J., Vandertweel, I., Herbschleb, J.N., Heethaar, R.M. and Borst, C. (1984) Nonrandom ventricular rhythm in horses with atrial fibrillation and its significance for patients. Journal of the American College of Cardiology 4, 316323.

[13] Summerfield, N. and Estrada, A. (2005) Ladder diagrams for atrial flutter and atrial fibrillation. Journal of Veterinary Cardiology 7, 131-135.

370

371

372

373

374

375

376

377

378

379

380

381

382

383

384

385

386

387

388

389

$390 \quad[19]$

391

392

393 [20]

394

395

396

$397 \quad$ [21]

398

399

400

$401[22]$

402

403

404
[14] Wallick, D.W., Stuesse, S.L. and Masuda, Y. (1986) Sympathetic and periodic vagal influences on antegrade and retrograde conduction through the canine atrioventricular node. Circulation 73, 830-836.

[15] Buber, J., Glikson, M., Eldar, M. and Luria, D. (2011) Exercise heart rate acceleration patterns during atrial fibrillation and sinus rhythm. Annals of Noninvasive Electrocardiology 16, 357-364.

[16] Langendorf, R. and Pick, A. (1956) Concealed conduction - Further evaluation of a fundamental aspect of propagation of the cardiac impulse. Circulation 13, 381-399.

[17] Langendorf, R., Pick, A. and Katz, L.N. (1965) Ventricular response in atrial fibrillation - Role of concealed conduction in the AV junction. Circulation 32, 69-75.

[18] Gelzer, A.R.M., Moise, N.S., Vaidya, D., Wagner, K.A. and Jalife, J. (2000) Temporal organization of atrial activity and irregular ventricular rhythm during spontaneous atrial fibrillation: An in vivo study in the horse. Journal of Cardiovascular Electrophysiology 11, 773-784.

Falk, R.H. and Leavitt, J.I. (1991) Digoxin for atrial fibrillation - A drug whose time has gone? Annals of Internal Medicine 114, 573-575.

Bootsma, B.K., Hoelen, A.J., Strackee, J. and Meijler, F.L. (1970) Analysis of R-R intervals in patients with atrial fibrillation at rest and during exercise. Circulation 41, 783-794.

Mendez, C., Gruhzit, C.C. and Moe, G.K. (1956) Influence of cycle length upon refractory period of auricles, ventricles, and A-V node in the dog. Am. J. Physiol. 184, 287-295.

Wiener, I., Kunkes, S., Rubin, D., Kupersmith, J., Packer, M., Pitchon, R. and Schweitzer, P. (1981) Effects of sudden change in cycle length on human atrial, atrioventricular and ventricular refractory periods. Circulation 64, 245-248. 
405 [23] Tadros, R. and Billette, J. (2009) Rate-dependent AV nodal refractoriness: a new

[24] Denes, P., Wu, D., Dhingra, R., Pietras, R.J. and Rosen, K.M. (1974) Effects of cycle length on cardiac refractory periods in man. Circulation 49, 32-41.

[25] Barbesgaard, L., Buhl, R. and Meldgaard, C. (2010) Prevalence of exercise-associated arrhythmias in normal performing dressage horses. Equine Vet. J. 42, 202-207.

[26] Buhl, R., Meldgaard, C. and Barbesgaard, L. (2010) Cardiac arrhythmias in clinically healthy showjumping horses. Equine Vet. J. 42, 196-201.

[27] Suyama, A.C., Sunagawa, K., Sugimachi, M., Anan, T., Egashira, K. and Takeshita, A. (1993) Differentiation between aberrant ventricular conduction and ventricular ectopy in atrial fibrillation using RR interval scattergram. Circulation 88, 2307-2314.

[28] Marriott, H.J.L. and Sandler, I.A. (1966) Criteria, old and new, for differentiating between ectopic ventricular beats and aberrant ventricular conduction in the presence of atrial fibrillation. Progress in Cardiovascular Diseases 9, 18-28.

Langendorf, R. (1951) Aberrant ventricular conduction. American Heart Journal 41, 700-707.

Nagayoshi, H., Janota, T., Hnatkova, K., Camm, A.J. and Malik, M. (1997) Autonomic modulation of ventricular rate in atrial fibrillation. American Journal of Physiology-Heart and Circulatory Physiology 272, H1643-H1649.

[31] Borer, K.E. and Corley, K.T.T. (2006) Electrolyte disorders in horses with colic. Part 1: potassium and magnesium. Equine Vet. Educ. 18, 266-271.

Borer, K.E. and Corley, K.T.T. (2006) Electrolyte disorders in horses with colic. Part 2: calcium, sodium, chloride and phosphate. Equine Vet. Educ. 18, 320-325.

[33] Zipes, D.P. (2008) Heart-brain interactions in cardiac arrhythmias: role of the autonomic nervous system. Cleveland Clinic Journal of Medicine 75, S94.

[34] Han, J., Dejalon, P.G. and Moe, G.K. (1964) Adrenergic effects on ventricualr vulnerability. Circulation Research 14, 516-524. 
446 [35] Engel, T.R., Meister, S.G. and Frankl, W.S. (1978) R-on-T phenomenon - Update and critical review. Annals of Internal Medicine 88, 221-225.

448

$449 \quad[36]$

450

451

452

453 [37]

454

455

$456[38]$

457

458

459

460

461

462
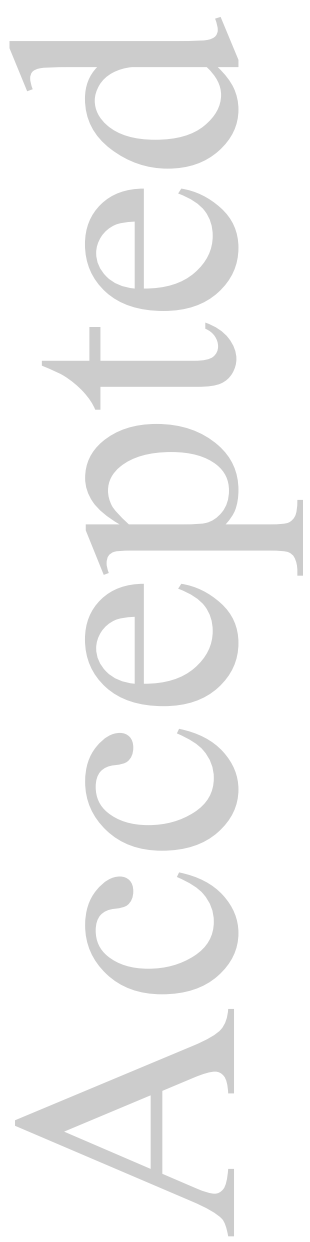

Lian, J., Muessig, D. and Lang, V. (2011) Risk Assessment of R-on-T Event Based on Modeled QT-RR Relationship. Pace-Pacing and Clinical Electrophysiology 34, 700708.

Lown, B. and Wolf, M. (1971) Approaches to sudden death from coronary heart disease. Circulation 44, 130-142.

Physick-Sheard, P.W. and McGurrin, M.K.J. (2010) Ventricular Arrhythmias during Race Recovery in Standardbred Racehorses and Associations with Autonomic Activity. Journal of Veterinary Internal Medicine 24, 1158-1166. 


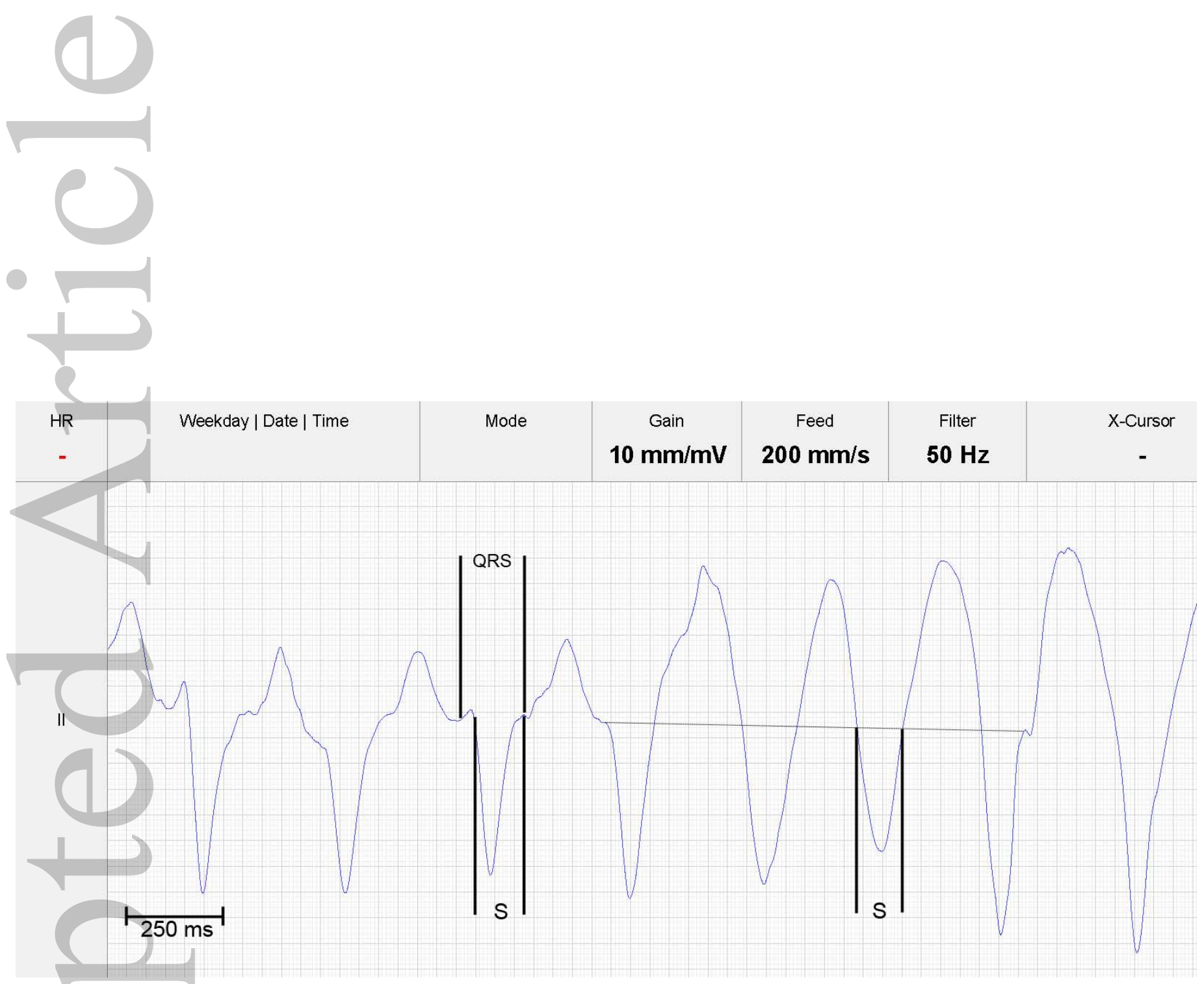

fig 1.tif 


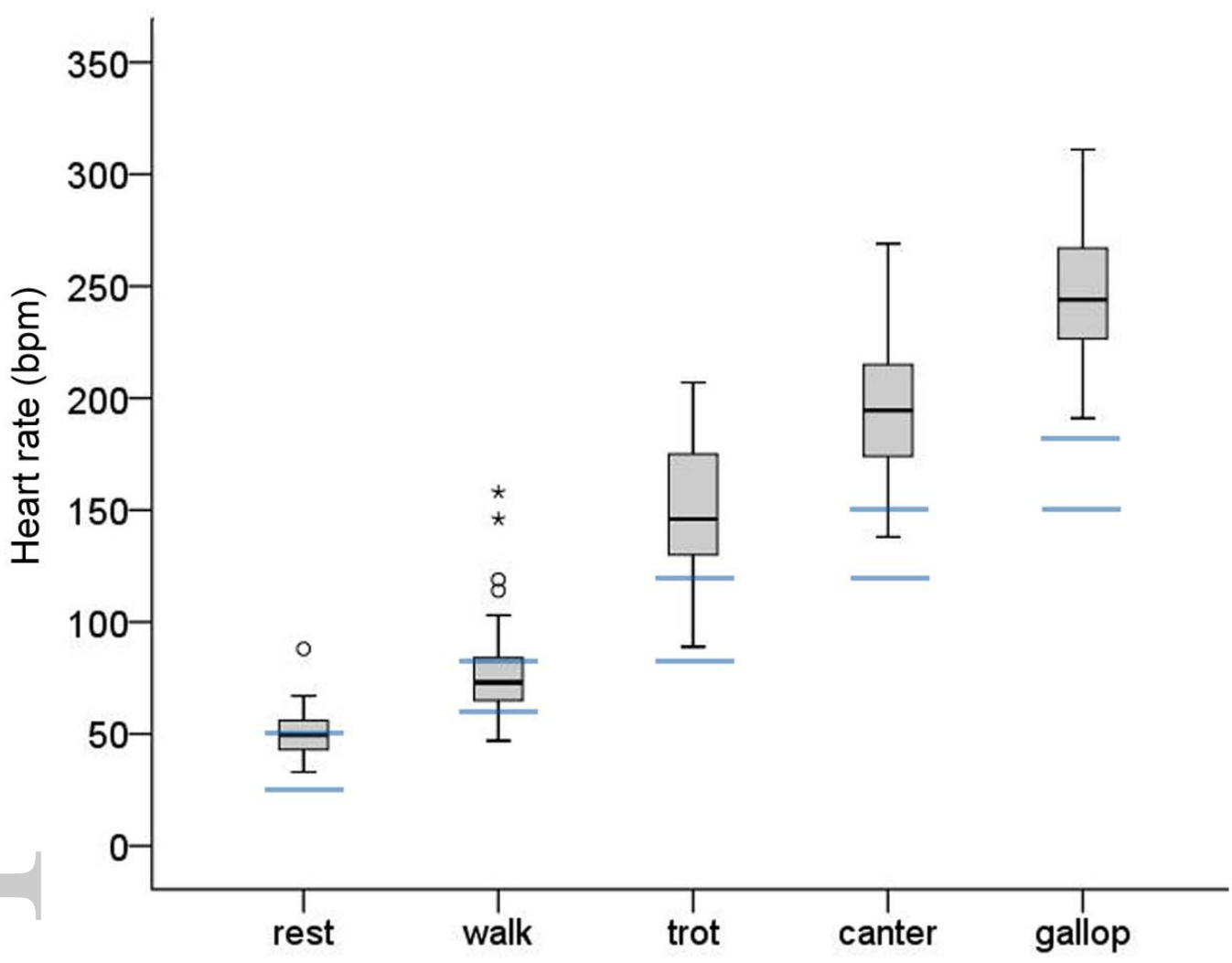

fig 2.tif 


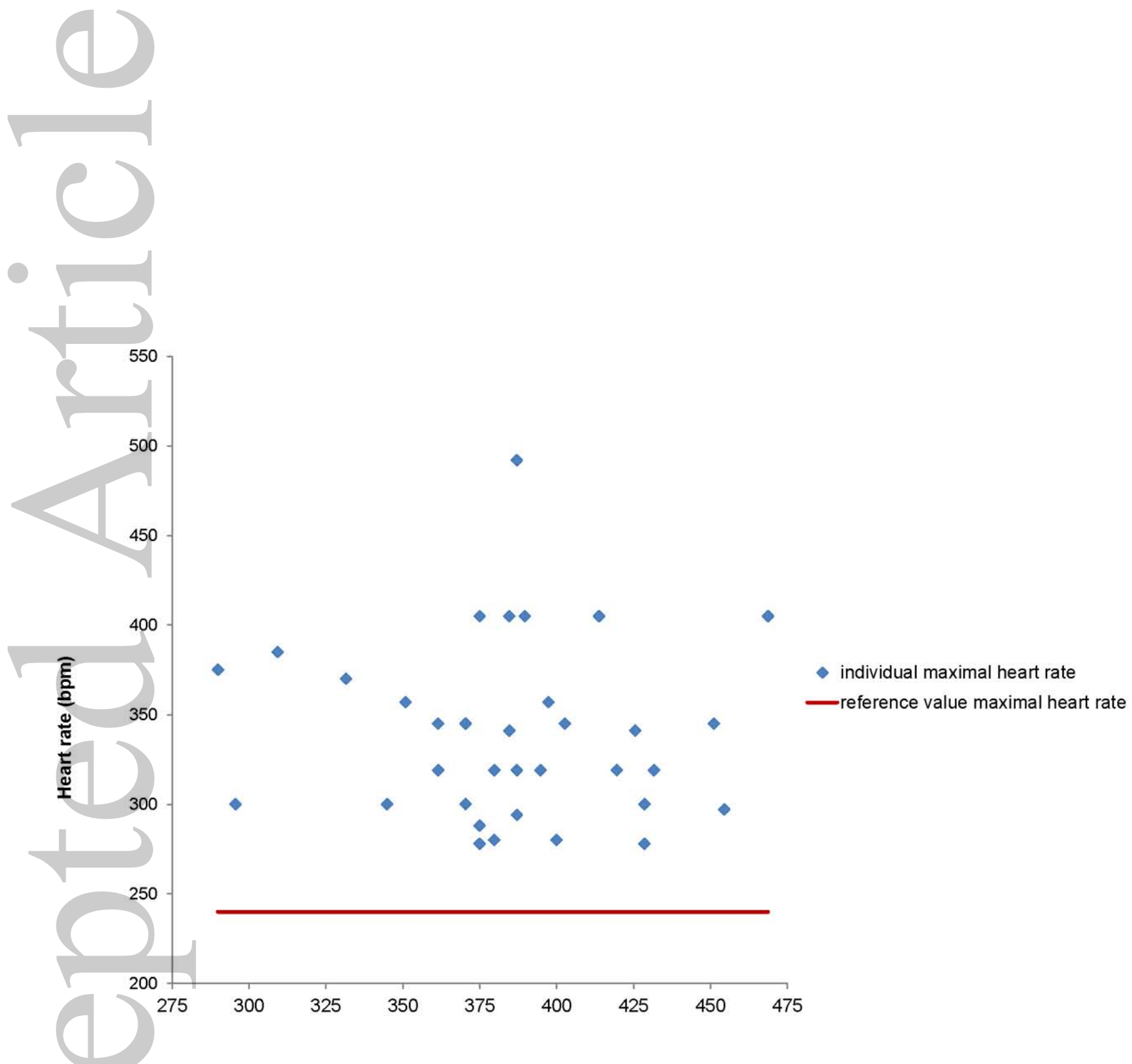

fig 3.tif 


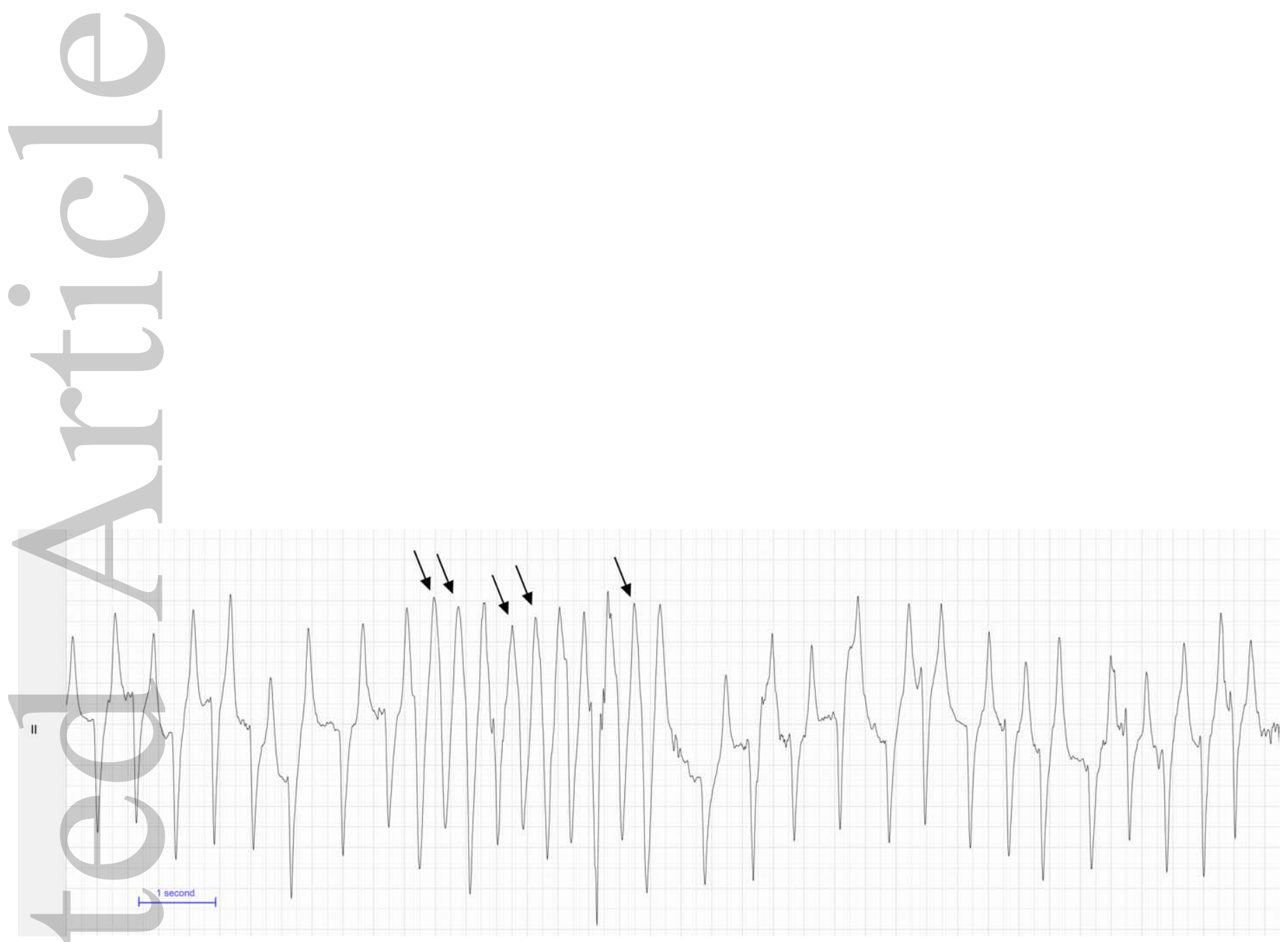

fig 4.tif

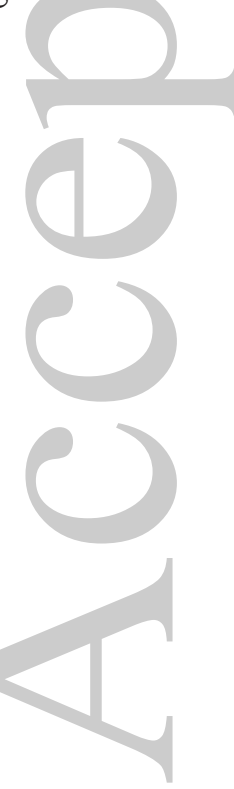




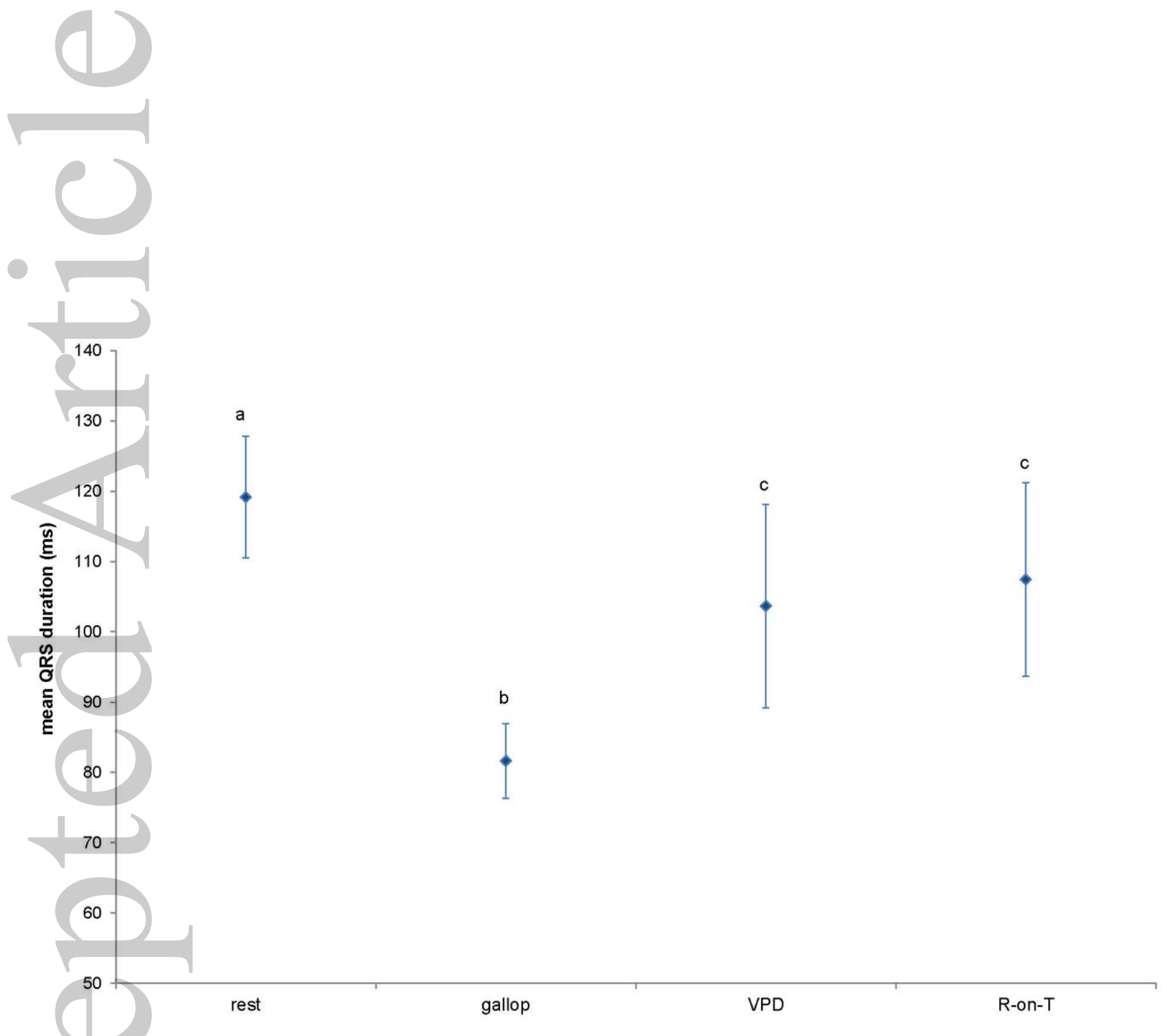

fig 5.tif 


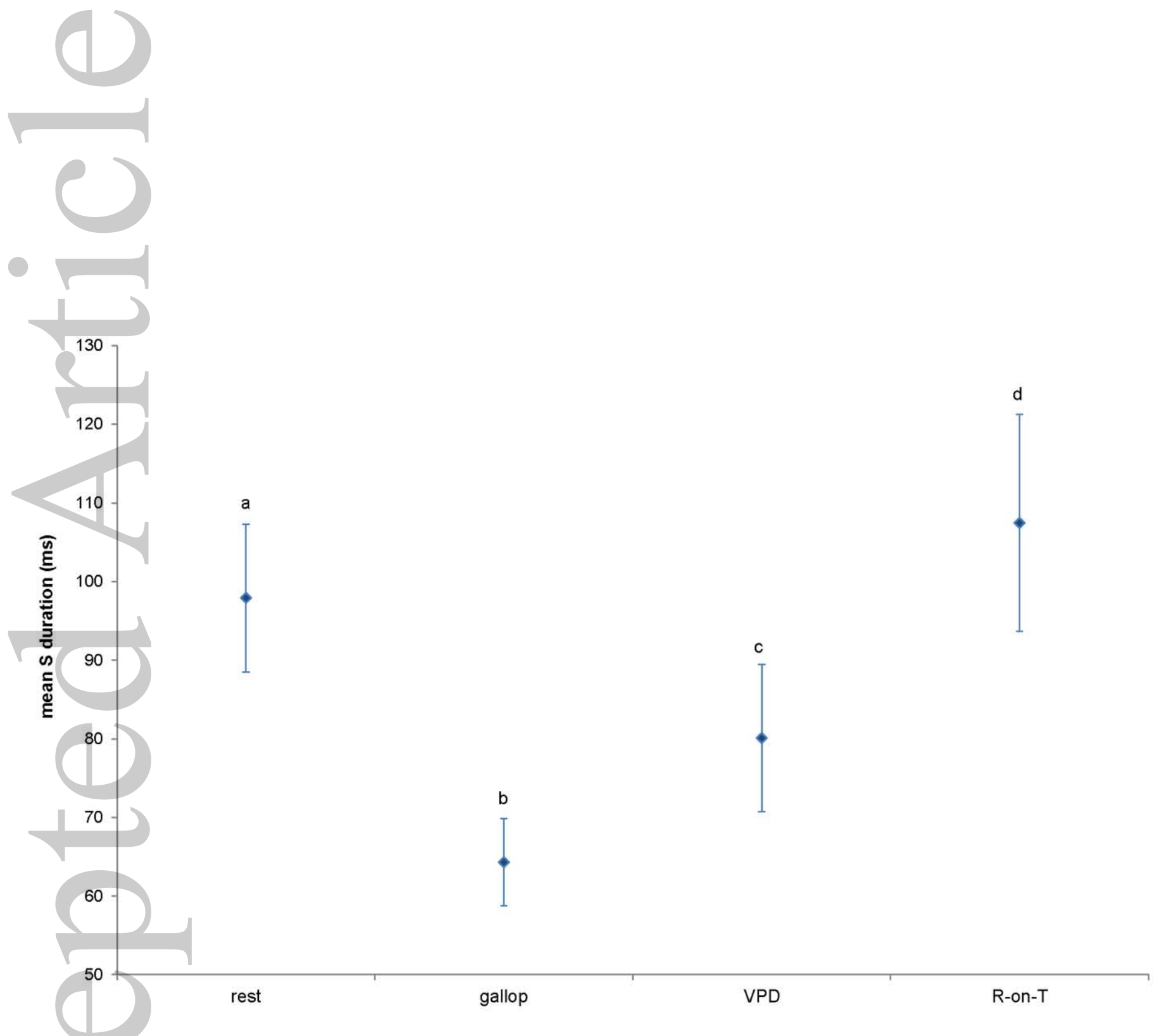

fig 6.tif 


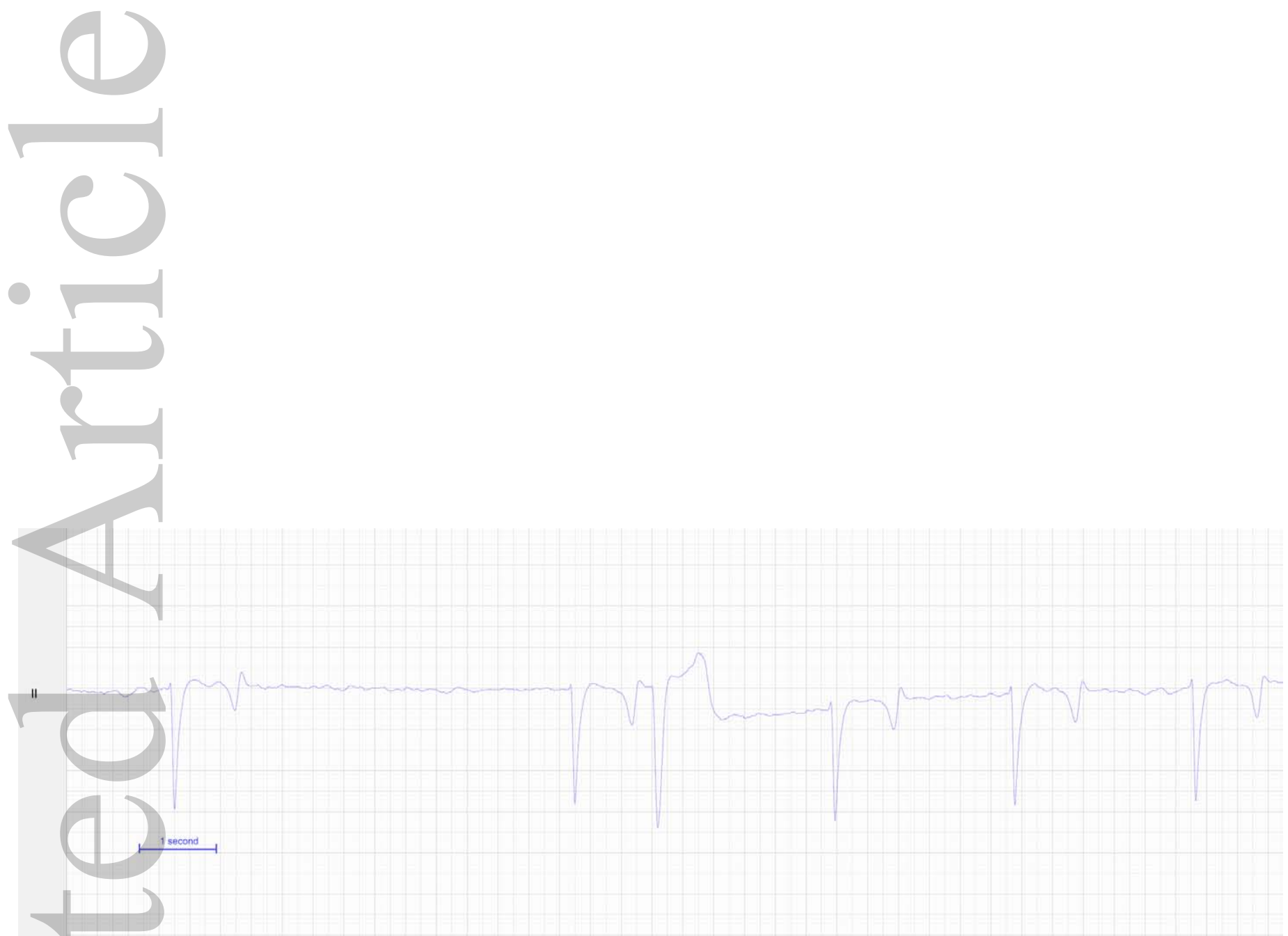

fig 7.tif

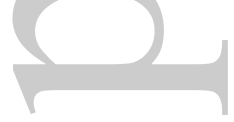

(c)
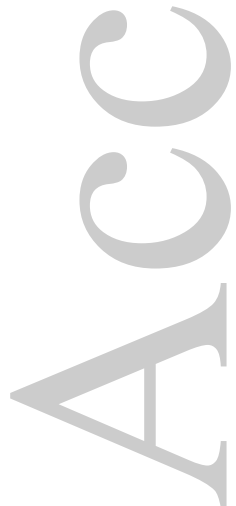